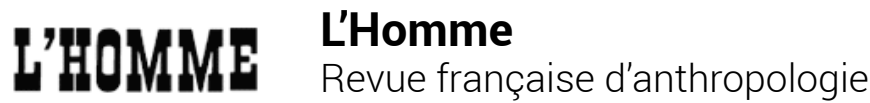

156 | octobre-décembre 2000

Intellectuels en diaspora et théories nomades

\section{Les Subaltern Studies ou la critique postcoloniale de la modernité}

Jacques Pouchepadass

\section{OpenEdition}

Édition électronique

URL : http://journals.openedition.org//homme/75

DOI : 10.4000/lhomme.75

ISSN : 1953-8103

Éditeur

Éditions de l'EHESS

\section{Édition imprimée}

Date de publication : 1 janvier 2000

Pagination : 161-186

ISBN : 2-7132-1348-7

ISSN : 0439-4216

Référence électronique

Jacques Pouchepadass, "Les Subaltern Studies ou la critique postcoloniale de la modernité », L'Homme [En ligne], 156 | octobre-décembre 2000, mis en ligne le 18 mai 2007, consulté le 30 avril 2019. URL : http://journals.openedition.org/lhomme/75 ; DOI : 10.4000/lhomme.75 


\title{
Les Subaltern Studies ou la critique postcoloniale de la modernité
}

\author{
Jacques Pouchepadass
}

L

ES SUBALTERN STUDIES sont une série de volumes collectifs publiée par Oxford University Press-Delhi depuis 1982, prévue au départ pour en comprendre trois, et qui à ce jour en compte dix. Les volumes portent le sous-titre Writings on South Asian History and Society. Le succès international de la série, alimenté par les débats théoriques et méthodologiques qu'elle a suscités dans le milieu de la recherche en sciences sociales, en Inde d'abord puis dans les pays anglo-saxons, a été exceptionnel pour un ensemble de recueils aussi spécialisés. Chacun des titres a connu plusieurs réimpressions, et l'édition de poche des dix volumes se vend depuis peu en bloc sous étui cartonné. Les volumes I à VI, publiés entre 1982 et 1989 , ont eu pour maître d'œuvre l'historien bengali Ranajit Guha (né en 1923), fondateur, inspirateur et animateur du collectif de six puis de dix chercheurs responsable de l'entreprise ${ }^{1}$. L'orientation intellectuelle initiale, d'ailleurs aussi politique que proprement théorique, était un marxisme critique dont les affinités se situaient du côté de Gramsci et d'historiens radicaux britanniques comme Edward P. Thompson. Guha a ensuite passé la main, laissant la direction des recueils suivants à des équipiers plus jeunes, sans cesser pour autant de collaborer au travail commun ${ }^{2}$. Les dix volumes parus, dont le niveau moyen est de très grande qualité, réunissent au total plus de quarante auteurs. La plupart de ces chercheurs, et en particulier les

1. Il s'agissait de Ranajit Guha, Shahid Amin, David Arnold, Gautam Bhadra, Dipesh Chakrabarty, David Hardiman, Gyanendra Pandey et Sumit Sarkar, historiens, et du politologue Partha Chatterjee, tous spécialistes de l'Inde moderne et travaillant en Grande-Bretagne, en Inde ou en Australie.

2. Le vol. VII (1992) est édité par Partha Chatterjee et Gyanendra Pandey; le vol. VIII (1994), soustitré Essays in Honour of Ranajit Guha, par David Arnold \& David Hardiman; le vol. IX (1996) par Shahid Amin \& Dipesh Chakrabarty; le vol. X (1999) par Gautam Bhadra, Gyan Prakash \& Susie Tharu. 
membres du collectif éditorial, ont publié par ailleurs d'autres travaux d'orientation identique, dont certains ont connu un incontestable retentissement ${ }^{3}$. Toute cette production a fini par constituer dans les sciences sociales indianistes un véritable courant, dont l'élan initial, comme on va le voir, est un peu retombé parce qu'il se dilue dans des courants plus larges, mais dont l'impact est désormais sensible parmi les chercheurs d'autres régions du Sud, notamment en Amérique latine ${ }^{4}$. Comme toutes les aventures intellectuelles collectives, celle-ci a été marquée par des divergences internes, des changements de cap, des sécessions, des ralliements, qui reflètent certains débats majeurs de l'épistémologie et des sciences sociales de ces vingt dernières années à l'échelle globale. Un membre fondateur comme Sumit Sarkar, un compagnon de route comme Ramachandra Guha, se sont désolidarisés du groupe et ont manifesté avec éclat leur désaccord avec le tour postmoderniste pris par la série à partir de la fin des années 80 (réorientation qui l'a propulsée sur la scène internationale des sciences sociales, mais lui a fait perdre une partie de son audience en Inde même). D'autres comme David Hardiman, qui font toujours partie du collectif, sont restés fidèles à une conception de l'histoire sociale qui n'est plus la ligne dominante de la série. L'analyse littéraire du discours et les études de "genre » (ce que la France appelle encore l'histoire des femmes) y ont fait une entrée en force, en même temps que le collectif accueillait de nouveaux membres (Gyan Prakash, Susie Tharu).

\section{Le paradigme de la conscience des dominés}

Le courant historiographique d'où les Subaltern Studies sont nées, c'est ce qu'il est convenu d'appeler «l'histoire par le bas" (history from below). Dans son principe, l'attitude intellectuelle qu'incarnait ce courant n'était bien entendu pas nouvelle: qu'on se rappelle l'introduction de la Révolution française de Michelet ("J'ai pris l'histoire en bas, dans les profondes foules, dans les instincts du peuple, et j'ai montré comment il mena ses meneurs, etc.»), et la résurgence régulière depuis lors de vocations individuelles ou collectives à écrire l'histoire du point de vue du plus grand nombre, qu'elles soient libérales, socialistes, nationalistes ou issues du courant des Annales. En Inde, les "passeurs» de cette orientation historiographique ont été, pour une part au moins, les ténors de l'histoire

3. Pour s'en tenir aux membres du collectif fondateur, on citera en particulier Amin 1995, Chatterjee 1986 et 1993, Hardiman 1987, Pandey 1990.

4. C'est d'ailleurs à une initiative africaniste que nous devons la publication récente en langue française d'un très utile recueil d'écrits théoriques de certains des principaux auteurs «subalternistes » indiens: Diouf 1999.

5. Le livre emblématique de ce courant est Thompson 1988 (1963). 
sociale "radicale» anglaise (Rodney Hilton, Christopher Hill, Georges Rudé, Edward P. Thompson, Eric Hobsbawm) ${ }^{5}$, à une époque (en gros à partir des années 60) où les désillusions suscitées en Inde par l'échec social de l'indépendance exacerbaient le radicalisme de la contestation marxiste dans les rangs de la jeunesse intellectuelle, et poussaient une fraction de celle-ci vers le militantisme maoïste dans les campagnes. L'idée qui se faisait jour alors parmi les historiens (en Inde comme dans d'autres aires de du monde anciennement colonisé), c'est qu'une véritable histoire par le bas impliquait une rupture avec le "paradigme nationaliste » de l'historiographie dominante, qui occultait les antagonismes de classes au nom de l'unité nationale, prolongeant ainsi la ligne politique officielle du combat d'indépendance ${ }^{6}$. C'est dans ce contexte de critique du marxisme orthodoxe et de la rhétorique socialisante officielle de l'État indien qu'est né, à l'initiative de Ranajit Guha, le projet des Subaltern Studies, expression intellectuelle d'une conception exigeante de la démocratie.

L'effort initial de Guha visait d'abord à en finir avec l'élitisme de l'historiographie colonialiste, nationaliste et marxiste, qui présentait la résistance populaire à la colonisation et l'épopée du mouvement d'indépendance comme le résultat d'un processus de mobilisation par le haut. L'historiographie colonialiste incriminée était avant tout une production anglo-saxonne des années 60 et 70 improprement désignée comme l'émanation d'une école (l'«école de Cambridge»), et universellement décriée aujourd'hui, qui présentait le nationalisme organisé en Inde comme une stratégie de promotion sociale maniée par les élites indigènes instruites afin d'arracher au pouvoir colonial des postes de responsabilité, générateurs d'honneurs et de profits. Selon la vulgate de l'histoire nationaliste de l'Inde moderne qui a régné à partir des années 50 dans les manuels et les grandes synthèses publiés sous patronage gouvernemental, le mouvement d'indépendance avait pris forme à travers un processus progressif d'englobement des masses populaires arriérées dans la lutte pour les libertés. Dans cette perspective, les grands intercesseurs de la politisation des masses étaient les leaders charismatiques, Gandhi et Nehru en tête, relayés vers le bas par la vaste structure d'encadrement panindienne qu'ils dirigeaient, l'Indian National Congress. Au sein du Congress confluaient toutes les couches sociales et presque toutes les sensibilités de l'opinion, unifiées dans la ferveur du combat commun pour l'émancipation et la naissance de l'État-nation. L'aboutissement logique de ce grand récit était l'accession au pouvoir dans l'Inde libre de ce leadership de la lutte d'indépendance et de l'organisation

6. Idée que formula très officiellement Sabyasachi Bhattacharya dans son allocution présidentielle d'ouverture devant la section d'histoire moderne de l'Indian History Congress de 1982 (Bhattacharya 1983). 
nationaliste devenue parti dominant. Ce dernier, qui reflétait dans sa propre diversité celle immense de la nation, était fondé à parler au nom du peuple tout entier, et sa tâche était désormais d'encadrer l'édification et le développement de la plus grande démocratie de la planète. Dans les études monographiques de mouvements populaires indiens parues au cours de années 70 et au début des années 80 se développaient néanmoins avec une insistance croissante des thèmes qui rompaient avec ces conventions narratives du nationalisme "bourgeois ". Il était de plus en plus clair que, tout en recherchant l'adhésion des masses pour se donner une crédibilité politique indispensable face au pouvoir colonial, l'élite nationaliste avait été constamment préoccupée par le souci de cantonner l'intensité et la radicalité de l'agitation populaire dans d'étroites limites, sachant que le message nationaliste congressiste et gandhien tel que les masses le décodaient et se l'appropriaient, en le peuplant de rêves millénaristes et de fantasmes d'inversion sociale, n'avait plus grand-chose à voir avec les conceptions et les normes policées de la démocratie représentative bourgeoise ${ }^{7}$. Ces études faisaient donc ressortir les failles qui lézardaient l'unanimité apparente de l'élan nationaliste populaire, la répression souvent exercée par le Congrès contre les déviations radicales de l'agitation, les malentendus profonds qui accompagnaient l'engagement des masses populaires, animées par leur culture originale de la contestation, au service des ambitions de l'élite qui s'arrogeait le privilège de les représenter. Ils donnaient ainsi une substance historique aux frustrations d'un peuple privé des bénéfices de sa victoire, une fois l'indépendance acquise, par la domination hégémonique du Congrès, organisation tentaculaire dominée par la classe moyenne urbaine et la paysannerie aisée, l'une et l'autre de haute caste, et dont la rhétorique socialisante masquait l'absence de toute volonté de réforme sociale radicale ${ }^{8}$.

L'historiographie marxiste du mouvement national, la seule qui tranchât vraiment sur le récit nationaliste conventionnel pendant les premières décennies de l'indépendance, péchait quant à elle par une autre forme d'élitisme. Tout en prétendant parler au nom des classes opprimées et pour éclairer leur marche vers l'émancipation et le progrès, elle stigmatisait leur culture propre de la résistance comme mentalité pré-politique ou fausse conscience, c'est-à-dire comme phase primitive du développement de la conscience révolutionnaire. Elle mettait en doute les capacités de lutte de la masse de la paysannerie, considérant que ses révoltes ne pouvaient être que des éclats de colère collective spontanés et sans lendemain, nécessaire-

7. Comme le montre, par exemple, Amin 1984.

8. Les meilleurs essais de synthèse de cette floraison des années 70 de l'historiographie critique du nationalisme indien sont Sarkar 1983a et 1983b. 
ment dépourvus d'organisation, de programme et d'efficacité aussi longtemps qu'ils n'étaient pas mobilisés et encadrés par une avant-garde mieux formée et politiquement plus avancée. Enfin, elle tombait dans le déterminisme économiciste en attribuant un rôle moteur décisif aux contradictions et aux conjonctures de crise de l'économie.

Il s'agissait donc de rétablir le peuple comme sujet de sa propre histoire en refusant de le concevoir comme simple masse de manœuvre manipulée par les élites, et en rompant avec les téléologies qui le transforment en agent passif d'une mécanique historique universelle (qu'il s'agisse de l'histoire nationaliste qui présente les révoltes de l'époque coloniale comme autant d'étapes dans la genèse de l'État-nation, ou de l'histoire marxiste qui les voit comme des stades de l'émergence de la conscience de classe). Il fallait reconnaître son importance historique réelle à la capacité d'initiative (agency) libre et souveraine de ce peuple, redécouvrir sa culture propre, s'intéresser enfin sérieusement à son univers de pensée et d'expérience (et pas seulement à ses conditions matérielles d'existence). Il fallait faire admettre en somme qu'il existe un domaine autonome de la politique du peuple distinct de celui de l'élite, dont les idiomes, les normes, les valeurs sont enracinés dans l'expérience du travail et de l'exploitation sociale. Le peuple, selon le manifeste programmatique publié par Ranajit Guha en tête du premier volume des Subaltern Studies, ce sont «les classes et groupes subalternes qui constituent la masse de la population laborieuse et les couches intermédiaires des villes et des campagnes", c'est-à-dire "la différence démographique entre la population totale de l'Inde et tous ceux qui en constituent l'élite ${ }^{9}$. Ce qui définit les subalternes (notion empruntée au Gramsci des Cahiers de prison), c'est la relation de subordination dans laquelle les élites les tiennent, relation qui se décline en termes de classe, de caste, de sexe, de race, de langue et de culture. Une certaine confusion s'est développée par la suite dans la série en raison du fait que la "subalternité » est définie tantôt comme une substance, comme la caractéristique discriminante d'une catégorie sociale particulière, et tantôt comme une relation (une élite indigène pouvant alors être considérée comme subalterne face à l'élite coloniale européenne). En calant son propos sur cette catégorie, Guha mettait en tout cas au centre de sa perspective historique une vision dichotomique de la société partagée entre dominants et dominés. Et s'il entreprenait de corriger la vision élitiste de l'histoire de l'Inde jusqu'alors prédominante, c'est au nom de la conviction que les élites indiennes exerçaient certes sur le peuple des subalternes leur domination (matérielle),

9. Guha 1982 : 4, et préface du même volume, p. VIII. Au lieu de « couches intermédiaires ", Guha parle un peu plus loin de "catégories inférieures de la petite bourgeoisie " (ibid. : 5). 
mais non pas leur hégémonie (c'est-à-dire leur suprématie culturelle) ${ }^{10}$. C'est ce domaine autonome de la pensée et de l'initiative des subalternes, systématiquement occulté par l'historiographie élitiste, qu'il fallait ressusciter, non seulement pour réparer l'injustice qui lui était faite et lui rendre sa dignité, mais pour exposer en pleine lumière le rapport de forces interne à un mouvement d'indépendance dont seules les élites avaient récolté les fruits, et pour éclairer, en vue des luttes futures, les raisons profondes de cet échec historique de la nation à réaliser sa destinée (" this historic failure of the nation to come into its own"), qui constitue le problème central de l'historiographie de l'Inde coloniale (Guha 1982: 7).

Pour rendre leur juste place à la conscience et à la capacité d'initiative des subalternes, qui étaient de culture orale et n'ont laissé pratiquement aucun témoignage sur eux-mêmes ${ }^{11}$, il n'y a selon Guha d'autre moyen que d'analyser les comptes rendus et rapports relatifs aux mouvements populaires que recèlent les archives coloniales, mais en les lisant à rebours ("against the grain", "à contre-fil »). Guha explique avec brio comment le discours de ces sources, avec lequel les historiographies élitistes sont par bien des côtés en consonance, tend en permanence à néantiser la conscience propre des subalternes en présentant les rébellions comme de simples réactions réflexes à l'oppression économique ou politique, dissolvant du même coup l'autonomie de vision et d'initiative des dominés dans la mécanique des causes extérieures ${ }^{12}$. Cependant, dans un livre magistral paru un an après le premier volume des Subaltern Studies (mais en chantier depuis une décennie), il montre tout ce qu'on peut tirer de ces sources produites par les élites du pouvoir quand on les décode à bon escient. Passant au crible les archives des révoltes paysannes survenues dans l'Inde coloniale entre 1783 et 1900 (c'est-à-dire avant la phase du nationalisme de masse), il met au jour dans cette étude, avec un grand luxe d'exemples, les caractères fondamentaux du comportement des révoltés, présentés en

10. Guha 1989. Le binôme hégémonie-domination est lui aussi emprunté à Gramsci, qui l'utilisait dans ses Cahiers de prison pour faire ressortir la différence de complexité de l'État en Occident et en Russie : la Révolution en Russie ne supposait que la conquête des structures de domination (administration, armée, police), qui constituaient tout l'État à elles seules; en Occident, la révolution doit l'emporter aussi sur l'hégémonie idéologique et sociale exercée par les élites sur la société civile, qui est elle-même au fondement du corps politique. L'application expéditive au cas de l'Inde coloniale de ce schéma déjà lourd de questions non résolues pour ce qui touche à l'Europe relevait évidemment d'une sociologie quelque peu hasardeuse. Sur le rapport des Subaltern Studies à Gramsci, voir Arnold 1984.

11. Rien d'équivalement, par exemple, au Moi, Pierre Rivière..., décrypté par Michel Foucault et son équipe (Foucault 1973), sauf rares exceptions comme les dépositions en justice des accusés du célèbre lynchage de policiers de Chauri Chaura, qui amena Gandhi à mettre fin à la campagne de non-coopération de 1920-1922, dépositions analysées dans Amin 1995.

12. Guha 1983a: 2-3 : "Insurgency is regarded as external to the peasant's consciousness and Cause is made to stand in as a phantom surrogate for Reason, the logic of consciousness " ( on situe le moteur de la révolte à l'extérieur de la conscience paysanne, et on substitue l'apparence de la Cause à la réalité de la Raison, à la logique de la conscience rebelle»). 
quelque sorte comme des invariants idéologiques de la conscience paysanne ${ }^{13}$. Ce faisant, il inaugure un travail neuf d'arpentage et de cartographie de ce continent autonome et indompté de la subjectivité des subalternes, qui n'est guère accessible à l'historien que sous les espèces d'une culture de la résistance à l'oppression (parce que les sources n'en parlent qu'à l'occasion des révoltes), et à travers le discours des oppresseurs. C'est cette voie que les auteurs des volumes ultérieurs des Subaltern Studies ont continué à défricher sous son impulsion.

Il très vite apparu, cependant, qu'une pareille entreprise était semée d'embûches théoriques. Le reproche le plus immédiat a été l'accusation de "mentalisme ": Ranajit Guha aurait été victime de l'illusion qu'il est possible de savoir ce qui se passe réellement dans la tête des gens (par exemple Stein 1985 : 2). C'était là se méprendre sur le sens profond de son propos, qui ne relevait pas prioritairement de l'histoire des mentalités, mais visait d'abord à critiquer l'herméneutique de l'historiographie dominante et l'occultation constante de la conscience et de l'expérience des subalternes qui en résultait, et à faire ressortir à quel point elle avait ainsi partie liée avec le pouvoir des élites. Plus sérieusement, beaucoup de critiques se sont étonnés de voir surgir, alors qu'on croyait l'humanisme bourgeois des Lumières enfin définitivement enterré, un programme historiographique axé sur la restauration du primat de la conscience et de l'autonomie du sujet (par exemple O’Hanlon 1988). La réponse subalterniste fut que cette démarche d'allure positiviste, qui tendait en effet à construire le subalterne comme sujet doté d'une intériorité propre, garante de son autonomie de pensée et de comportement, était en fait une stratégie concertée à visée plus politique que théorique (Spivak 1985: 342) : il s'agissait d'en finir avec le métarécit élitiste de l'unanimité sociale du mouvement nationaliste, qui sert de mythe d'origine à l'État bourgeois de l'Inde indépendante, en montrant que l'élite avait construit sa victoire sur la négation de son Autre, le peuple des dominés. Le problème n'en est pas moins réel parce que tous les auteurs de la série, incontestablement, n'ont pas mis cette stratégie en œuvre avec autant de subtilité que Ranajit Guha. Le risque majeur était bien sûr d'essentialiser la conscience subalterne comme une nature, définie en dehors de tout contexte par un ensemble de traits distinctifs, ou pire, de réifier la catégorie de "subalterne " en l'assimilant à une entité sociale empirique ${ }^{14}$. Or, du point de vue sociologique, une telle

13. Guha 1983b. Ces «formes élémentaires" sont analysées sous les rubriques suivantes : négation (la conception populaire de la résistance), ambiguïté (le rapport entre délinquance et résistance), modalité (les formes d'organisation et d'action collective), solidarité (unité et discordances dans la rébellion), transmission (la rébellion comme fait de communication), territorialité (l'horizon spatial des rébellions). 14. À l’image de Stephen Henningham (1983), qui décrit le mouvement «Quit India » de 1942 comme .../... 
catégorie, qui englobe de fait les classes populaires dans leur ensemble, est tellement vaste et recouvre tant de nuances, de clivages, de relations de pouvoir internes au monde des dominés qu'elle est inutilisable comme outil d'analyse. Toutefois, la représentation dichotomique parfaitement simpliste du social qu'elle implique fait sens politiquement, en tant qu'elle exprime le rapport de pouvoir qui constitue historiquement le subalterne en tant que tel, c'est-à-dire la situation qui oppose, dans un contexte donné, l'élite et l'ensemble des dominés: cette situation se ramène en effet, du point de vue du subalterne, à une relation antagonique entre « nous » et « eux ». Mais le glissement vers l'essentialisation des subalternes était tout aussi difficile à éviter entièrement que l'était l'essentialisation de la classe dans le discours marxiste traditionnel. Beaucoup de contributeurs aux Subaltern Studies ont cédé à cette dérive, et l'on ne saurait dire que Ranajit Guha lui-même y ait totalement échappé.

Les Subaltern Studies de cette première phase, celle qui développait directement la problématique marxiste dissidente initialement tracée par Ranajit Guha, ont également eu à subir tout un faisceau de critiques venues d'autres courants du marxisme ${ }^{15}$. Face à la critique subalterniste de l'élitisme de l'historiographie dominante, on a vu se profiler en filigrane la vieille accusation de populisme, qui servait jadis à stigmatiser comme hérétiques ceux qui refusaient de reconnaître aux éléments d'avant-garde leur rôle moteur dans les luttes sociales. Moins aisées à récuser, en revanche, étaient les objections dirigées contre la définition intransigeante de l'autonomie de conscience et d'initiative des subalternes, qui est vite devenue un des axiomes de la série. Le point de départ de cette attitude était le refus par Guha de l'historiographie conventionnelle qui limitait l'univers de la politique au champ étroit des institutions coloniales dans lequel s'inscrivait l'activité contestataire des élites. Il opposait à ce domaine de la politique bourgeoise celui de la politique du peuple, ensemble de représentations et de pratiques de résistance enraciné dans un vieux passé précolonial, qui ne devait rien à ce sous-produit de la colonisation qu'était la politique de l'élite, et qui s'en distinguait par ses processus originaux de mobilisation non pas verticaux mais horizontaux (car fondés sur la parenté, le territoire, les solidarités de condition), par son caractère plus spontané, par le recours plus fréquent à l'action violente. Un marxiste orthodoxe pouvait à la rigueur suivre Guha jusque-là, quoique en dévalorisant cette politique du peuple comme inefficace et objectivement réac-

une révolte «duelle», soulèvement organisé de l'élite nationaliste d'un côté, rébellion violente de subalternes animés d'une conscience religieuse "caractéristique " de l'autre.

15. Voir par exemple les comptes rendus collectifs des Subaltern Studies parus dans Social Scientist en octobre 1984 et mars 1988; Mukherjee 1988; Brass 1993. 
tionnaire. Mais Guha allait plus loin. Il ne se contentait pas de dénoncer la manœuvre par laquelle l'élite nationaliste, au cours de la lutte anticoloniale, avait instrumentalisé l'élan de la contestation populaire à son profit exclusif afin d'exercer le pouvoir sans partage une fois l'indépendance acquise. Il présentait cette politique du peuple comme une expression du domaine autonome de cette conscience subalterne toujours réfractaire à l'emprise de la bourgeoisie, un élément de ces «vastes zones de la vie et de la conscience du peuple qui n'ont jamais été intégrées dans son hégémonie» (Guha 1982: 4-5), donnant ainsi la mesure à la fois de l'injustice sociale sur laquelle l'indépendance s'était historiquement construite, et de la nécessité de luttes futures.

Il y avait là manifestement l'amorce d'un glissement du constat, incontestable quoique souvent minimisé ou occulté par l'historiographie, de l'existence de poussées conjoncturelles autonomes de résistance des subalternes lors du combat d'indépendance, à la suggestion beaucoup plus lourde d'implications de l'autonomie structurelle de la conscience et de la culture subalternes. On peut supposer que chez Guha, cette intuition était avant tout affaire d'empathie vis-à-vis de l'imaginaire des révoltés et de visée politique, deux dispositions d'esprit qui peuvent l'une et l'autre légitimement fonder une lecture historienne des sources du moment qu'elles sont clairement affichées au départ. C'est en tirant cette intuition dans le sens du système que les historiens subalternistes se sont créé des difficultés théoriques difficilement surmontables. Ils prenaient par là position sur le terrain des modèles des sciences sociales, posture peu conforme à la vocation avant tout critique de l'histoire, avec pour tout bagage l'évidence empirique de la répression nationaliste des courants plébéiens pendant le mouvement d'indépendance, et leur impatience politique d'intellectuels de gauche qui en découlait aujourd'hui. Ce «modèle» subalterniste manquait évidemment de substance sociologique et anthropologique, et semblait revenir au point de départ de décennies de travaux et de controverses, pour une part déjà presque oubliés, sur le statut de la culture populaire, sur le rapport entre "grande» et "petite» tradition, sur l'acculturation. Il ne faisait pas un sort clair à la caste, pourtant point focal de la recherche sociologique sur l'Inde pendant la plus grande partie du siècle écoulé. Récusant, comme économiciste et téléologique, l'analyse de classes des mouvements populaires, certains des subalternistes au moins préféraient voir dans la " communauté » le cadre naturel du sentiment d'identité et de la mobilisation des paysans - retournant ainsi apparemment à la notion marxienne de la communauté comme cadre «naturel» de la vie sociale dans les sociétés antérieures à la classe (entre autres Chatterjee 1988 : 1014). À la limite, on avait moins affaire dans certains cas à une sociologie 
qu'à une doctrine sociale, avec ce que cette notion implique de postulats militants et d'orientation vers l'action.

La critique opposée par les historiens marxistes aux Subaltern Studies était incontestablement pertinente sur plusieurs points. Prenant acte du fait que les subalternistes refusent toute détermination extérieure de l'action des subalternes, et notamment tout élément d'explication économique des soulèvements (démarche invariablement taxée de déterminisme économiciste), elle leur reprochait de négliger l'étude des causes et du contexte des mouvements, pour ne s'intéresser qu'aux formes de la contestation populaire. C'était en effet défendre une notion anhistorique du "domaine autonome» des subalternes, et ignorer les transformations de la contestation populaire d'une époque à l'autre (pouvait-on admettre par exemple que la contestation paysanne n'ait pas évolué entre l'époque précoloniale et l'époque coloniale, alors que la colonisation avait transformé le contexte de pouvoir ?). Les subalternistes tendaient par ailleurs à caractériser la résistance populaire comme violente (la lutte non violente étant vue comme élitaire par nature), alors que les modes de résistance varient en fonction du type de pouvoir ou de répression à combattre, et que la résistance la plus violente n'est pas nécessairement la plus révolutionnaire. Ils faisaient preuve d'une sorte de cécité face à l'évidence massive que le leadership des mouvements populaires pouvait être assuré par des nonsubalternes, ou à tout le moins par des subalternes dotés de connexions avec la société englobante (évidence peu compatible avec la thèse de l'autonomie). Ils refusaient de reconnaitre que l'idéologie des rébellions subalternes pouvait être conservatrice, visant non pas à la subversion de l'ordre coutumier mais plutôt, quand cet ordre était menacé ou compromis, à sa restauration. Enfin nul ne peut se satisfaire, face à la question complexe des formes et des degrés d'interaction idéologique et culturelle entre les élites et les classes populaires, de l'assertion pure et simple de l'autonomie de la conscience et de la culture des subalternes. Ajoutons que si l'on attribue aux subalternes, comme le fait Guha (1982: 5), une idéologie dont "l'un des traits invariants était une notion de résistance à la domination de l'élite", on ne voit plus comment rendre compte des attitudes de consentement des dominés à la domination qu'ils subissent, qui sont pourtant beaucoup plus permanentes parmi les subalternes, en Inde comme ailleurs, que les comportements de résistance ${ }^{16}$.

16. Attitudes dont traitait La Boétie dans son Discours de la servitude volontaire, et dont beaucoup d'auteurs ont reparlé plus récemment, de Moses Finley à propos de l'esclavage dans la Grèce antique à Claude Lévi-Strauss, Pierre Clastres et Maurice Godelier dans de tout autres contextes. 
C'étaient là de vraies difficultés. Il faut dire cependant que les études réunies dans les six premiers volumes des Subaltern Studies (ceux dont Guha lui-même était le maître d'œuvre) n'en représentaient pas moins pour la plupart du travail historiographique d'excellente facture. Les textes fondateurs de Ranajit Guha ${ }^{17}$ font partie de ces énoncés programmatiques inspirés qui esquissent un questionnaire pour une génération d'historiens. La richesse, la nouveauté et la solidité du travail sur les archives qui ont nourri ces volumes sont tout aussi incontestables que la cohérence de ce travail avec les interrogations politiques des intellectuels indiens radicaux à partir des années 60 . À quelques notables exceptions près, toutefois, les contributions individuelles étaient beaucoup moins nettement "problématisées " que les textes inauguraux de Guha, et il a fallu plusieurs années et la parution de plusieurs volumes de la série pour que se dessinent sous la plume des critiques des vues d'ensemble faisant explicitement ressortir les difficultés théoriques qu'on vient d'exposer. Les membres du groupe subalterniste eux-mêmes, d'ailleurs, considéraient plutôt leur collectif comme un forum de discussion que comme une entité professant une doctrine homogène ${ }^{18}$. À ce stade, c'est-à-dire vers la fin des années 80 , le groupe ne s'en trouvait pas moins devant une alternative. Ou bien il poursuivait l'inventaire descriptif des formes de l' "insurgency" subalterne en conformité avec sa visée politique initiale. Il courait alors le risque d'une production quelque peu répétitive, et se devait d'affronter le problème des dérives essentialistes relevées par les critiques, et donc d'affiner sa façon de traiter de l'autonomie de conscience et d'action des subalternes, qui constituait le cœur du projet initial. Ou bien il changeait de registre, abandonnant le dessein de décrire dans sa positivité (d'ailleurs hypothétique) ce domaine autonome de la conscience et de l'expérience des subalternes, et reportait son effort principal sur la critique du paradigme élitiste des sources et de l'historiographie. De l'effort militant de résurrection de la culture et de l'expérience du peuple, on passait dans ce cas à un travail de déconstruction du discours des élites. On restait par là dans le prolongement de l'un des axes de recherche inaugurés par Guha, la lecture «à contre-fil» de la "prose de la contre-rébellion»(Guha 1983b). Mais, nuance capitale, ce qui n'était chez Guha qu'une technique de décryptage des textes au service d'une recherche politiquement finalisée devenait alors le point focal de la recherche elle-même.

17. C'est-à-dire essentiellement sa contribution à Subaltern Studies I (Guha 1982) et l'Introduction de son livre paru peu après (Guha 1983a).

18. Hardiman 1986. D. Chakrabarty écrivait en 1985 : « [les membres du groupe subalterniste] ne sont pas une "secte" dotée d'un point de vue unique ; ils sont sans doute beaucoup plus unis dans leur rejet de certaines positions et tendances universitaires que dans l'adhésion à des alternatives simples, quelles qu'elles soient»(Chakrabarty $1985: 364)$. 


\section{Le tournant postmoderniste}

172 C'est cette seconde orientation, politiquement moins engagée peut-être, mais plus conforme à l'esprit nouveau des sciences sociales, qui a prévalu dans la série à partir de la fin des années 80 . Il faut dire que certaines formulations critiques de Guha, qui étaient issues comme on l'a dit du terreau de l'histoire par le bas des historiens radicaux des années 60, avaient commencé à prendre au cours des années 80 , dans l'esprit d'une partie au moins de ses jeunes collègues (car il y avait vingt-cinq ans d'écart entre Guha et l'âge moyen des membres du collectif subalterniste), une résonance intellectuelle inédite. Ce n'est pas ici le lieu d'évoquer dans sa généralité, puisqu'il en est abondamment question ailleurs dans ce numéro, l'influence qu'exerçaient déjà sur une fraction des intellectuels indiens, et notamment de ceux qui fréquentaient le plus assidûment les universités américaines, la critique ambiante de la rationalité et des errements du progrès, et le relativisme épistémologique qui souvent la prolongeait. Ces courants de pensée, et notamment l'idée qu'on tirait de Michel Foucault que la vérité, naguère encore conçue comme réalité transcendantale, a une histoire empirique indissociable du champ social et politique occidental, et plus généralement la grande thématique foucaldienne du complexe pouvoir-savoir, prenaient évidemment une signification particulière dans l'optique de ce qu'on commençait à appeler le paradigme postcolonial. Edward Said, avec son Orientalism, essai d'archéologie du discours occidental sur l'Orient, paru trois ans après Surveiller et punir de Foucault, avait fait ressortir avec force que représenter l'autre c'est le manipuler, que le concept qui essentialisait l'oriental à l'âge de l'expansion européenne avait été instrument de soumission ${ }^{19}$. C'est principalement par son entremise que la nébuleuse de pensée critique qu'on qualifie globalement de postmoderniste a commencé à infiltrer l'historiographie indienne, avec les Subaltern Studies comme point de fixation principal.

Son effet le plus évident a été le déplacement de la critique du colonialisme du champ économique et politique au champ culturel. Du constat de Guha relatif à l'échec de l'État moderne à exprimer la vérité de la nation, à l'heure où Benedict Anderson (1983), dans un livre qui cristallisait très habilement certaines questions du moment, mettait en relief la part de l'imaginaire dans les mouvements nationaux, on glissait au diagnostic général d'échec de la modernité. «La culture bourgeoise », écrivait

19. Au prix d'une certaine essentialisation de l'orientalisme lui-même, et sans dire ce que pourrait être une compréhension moins mutilante, sans faire la part non plus de la contribution concertée de certaines élites indigènes à l'édification du savoir orientaliste (idée à laquelle un essai de Bernard S. Cohn (1985: 284 et 329) paru dans Subaltern Studies IV faisait fugitivement allusion. 
R. Guha (1989), "a atteint sa limite historique avec le colonialisme. Aucune de ses nobles conquêtes - le Libéralisme, la Démocratie, la Liberté, le Règne de la Loi, et ainsi de suite - ne peut survivre à l'inexorable nécessité inhérente au capital de s'étendre et de se reproduire en menant hors de ses frontières une politique de domination coloniale." Dans le nouveau contexte intellectuel, cette dénonciation, pourtant aussi vieille que l'anti-impérialisme lui-même, s'est mise à jouer le même rôle pour au moins une partie des subalternistes que les grandes régressions barbares de l'Europe du $\mathrm{XX}^{\mathrm{e}}$ siècle pour nombre de penseurs occidentaux : elle fonctionne comme un rouage majeur dans la critique de la légitimité du "métarécit » des Lumières et du paradigme européocentrique du progrès. Elle figurait certes déjà au premier rang dans le discours d'émancipation du nationalisme comme du marxisme. Mais l'un et l'autre partageaient la vision originaire (foundational) de la Raison comme forme universelle et de l'Europe comme moteur historique de l'odyssée du Progrès. C'est ce dispositif métanarratif lui-même qui est maintenant en cause. Il y avait, affirme D. Chakrabarty (1991 : 2163), de la «naïveté théorique " de la part des subalternistes à emboîter le pas aux grand discours d'émancipation modernes comme ils l'ont fait au départ. Il s'agit désormais de faire comprendre que la raison n'est qu'un modèle culturel parmi d'autres, et par là-même de "provincialiser " l'Europe, ce référent théorique implicite de toutes les historiographies élitistes ${ }^{20}$. Il s'agit même éventuellement de la "tiermondiser", en soutenant et en stimulant la résistance des subalternes du monde développé ${ }^{21}$. Il faut restaurer la différence culturelle dans sa dignité, en finir par exemple avec le terrorisme interprétatif qui stigmatise toute solidarité non assignable à la conscience de classe comme fausse conscience, réhabiliter les «temps ethniques non disciplinés par l'État et l'écriture » ${ }^{22}$, restituer les représentations identitaires, les mémoires, les voix populaires occultées ou opprimées d'abord par la domination occidentale, puis par le récit de l'historiographie dominante complice de la modernité.

Le lieu où le nouveau cours des Subaltern Studies prend sa source est donc bien identifié : c'est l'intersection du postmodernisme et de la postcolonialité. Le fait qu'un tel projet ait ainsi d'abord pris racine dans le domaine indien n'est sans doute pas un hasard. L'Inde est certainement la région du monde colonisé où un enseignement supérieur étroitement encadré par la métropole a fonctionné de la façon la plus précoce et à

20. Chakrabarty 1992 (article repris en traduction in Diouf 1999).

21. Prakash 1990b: 403 (également repris in Diouf 1999).

22. Diouf 1999 : 24, d'après une formule de Jacques Le Goff. 
l'échelle la plus ample, où le discours historique de la modernité, du capitalisme et de la démocratie a tissé les liens les plus forts, et dont les connexions avec les milieux universitaires occidentaux sont les plus étroites. Ses chercheurs étaient du même coup particulièrement bien placés pour opérer ce genre de retour critique sur les discours métropolitains (Prakash 1994 : 1489). On comprend aussi pourquoi la série a accédé lors de cette deuxième phase à une notoriété internationale qui la pose en véritable référence pour au moins une fraction de l'establishment universitaire d'Outre-Atlantique ${ }^{23}$ : c'est qu'elle donne à la mouvance postmoderniste un enracinement extra-occidental, en même temps qu'elle commence à faire école dans d'autres régions du Sud, et notamment en Amérique latine, où les malentendus et contradictions entre mouvements nationalistes et couches subalternes présentent des analogies avec le cas indien. Mais simultanément, l'intensité du débat suscité en Inde même par la parution des premiers volumes de la série est pour une bonne part retombée (Sarkar 1997: 84).

Ce qui est perçu de l'extérieur comme une réorientation est plutôt conçu par la majorité des membres du projet subalterniste eux-mêmes comme le moment où le groupe, après une période initiale de débat interne très ouvert, a fini par trouver sa cohérence autour d'une problématique plus strictement nouée. Il était clair que la focalisation sur la conscience subalterne (plutôt que sur les conditions d'existence ou l'histoire des luttes des subalternes dans leurs contextes régionaux particuliers), qui était la marque distinctive du projet, débouchait sur une impasse s'il s'agissait de reconstruire le subalterne en tant que sujet indépendant du discours de l'élite. Il fallait donc privilégier l'autre terme de l'alternative, et reconnaître enfin définitivement que la subalternité, souvent prise par erreur pour une catégorie sociologique, n'est pas une substance mais une relation, et n'a d'existence qu'en tant qu'elle est constituée par le discours de l'élite (coloniale et nationaliste) comme force de résistance à l'hégémonie de celle-ci. Ce thème courait déjà dans un livre de Partha Chatterjee paru en 1986, Nationalist Thought and the Colonial World. Cet essai montrait que le discours nationaliste de l'élite indienne, certes opposé au discours colonial mais enraciné dans le même fonds idéologique, celui de la modernité bourgeoise, était confronté à une contradiction fondamentale: l'élite se devait de parler au nom des masses si elle voulait affronter le pouvoir colonial avec une chance de succès, mais elle redoutait en même temps, et contenait avec peine, l'irrépressible autonomie de leur mode de contestation. La tâche des historiens

23. Notoriété puissamment assistée par la publication aux États-Unis, sous la direction de Ranajit Guha et Gayatri Spivak, d'une anthologie des Subaltern Studies précédée d'un avant-propos d'Edward Said (Guha \& Spivak 1988). 
subalternistes, c'était donc avant tout la déconstruction du discours dominant et normalisateur des textes coloniaux et nationalistes pour en faire apparaître le refoulé, c'est-à-dire la culture, l'expérience, la mémoire systématiquement élidées du peuple. Un tel travail, sans doute, exposait l'historien à une tension permanente entre le fait que la subalternité est avant tout un effet de situation et la tendance difficilement évitable à la représenter comme une qualité intrinsèque. Il n'en était pas moins clair que le projet ne visait pas prioritairement à proposer un nouveau modèle de stratification sociale (qui serait d'ailleurs simpliste), mais à réintégrer le pouvoir comme donnée centrale de l'organisation sociale, et à démontrer que la vision libérale bourgeoise de l'État-nation comme entité plurale mais consensuelle est fondamentalement fausse.

L'inconvénient de cette polarisation sur la "textualité " du colonialisme et du nationalisme, sur la critique du discours des élites, c'est qu'elle dématérialisait définitivement le domaine de l'antagonisme entre ces dernières et les subalternes pour le confiner dans l'ordre de la culture. La distance prise dans le même mouvement par rapport à ce qu'on étiquetait comme le modèle universaliste occidental de la rationalité conduisait à valoriser la différence pour elle-même, indépendamment de tout autre critère normatif que la résistance à l'emprise uniformisante de la modernité. Insensiblement, beaucoup de subalternistes passent ainsi de l'opposition binaire élite/subalternes à l'opposition modernité occidentale/culture indigène. Ils tendent à réhabiliter en bloc le précolonial au nom de la critique du colonial, ou (s'agissant de l'Inde contemporaine) le minoritaire et le marginal au nom de la critique de l'État-nation né du combat d'indépendance, incarnation en Inde d'une modernité politique oppressive qui remonte à l'idéologie des Lumières. Gyan Prakash (qui a récemment rejoint le collectif éditorial des Subaltern Studies), analyse, par exemple, dans un livre brillant paru en 1990, Bonded Histories: Genealogies of Labor Servitude in Colonial India, la façon dont la relation entre les paysans dominants de la région gangétique du Bihar et les dépendants qu'ils emploient comme ouvriers agricoles a évolué depuis l'époque coloniale. La distinction entre employeurs et ouvriers, dit Prakash, ne faisait autrefois que matérialiser une différence de statut propre à la société hiérarchique des castes. Ce partage fonctionnel impliquait de part et d'autre des droits et des devoirs englobant tous les aspects de l'existence, et incluait notamment des relations de crédit, qui n'étaient qu'un aspect annexe du rapport employeur-employé et non pas son fondement. C'est dans le sillage de l'abolition de l'esclavage en Inde au nom de l'idéal libéral hérité des Lumières que les Britanniques, au XIX ${ }^{\mathrm{e}}$ siècle, ont requalifié juridiquement cette relation de dépendance pour la conformer au droit moderne et la 
rendre du même coup susceptible de correction par les voies judiciaires ordinaires. Ils l'ont alors assimilée à un engagement contractuel de travail souscrit en contrepartie d'une fourniture de crédit. Or cet endettement qui pèse sur les plus pauvres des pauvres s'avère presque toujours impossible à rembourser étant donné l'exiguïté extrême de leurs revenus. Les Britanniques, par leur réforme, ont donc effectivement institué la servitude pour dettes, et fait de l'ouvrier agricole un non-libre. Tous les efforts ultérieurs de l'État pour abolir cette forme de servitude ont échoué jusqu'à aujourd'hui, et la violence de cette relation employeur-employé réduite à sa composante économique est devenue inexpiable.

La force d'impact de ce type de critique de la modernité ne vient pas de ce qu'il la fait apparaître comme incomplète ou insuffisamment efficace, mais qu'il la révèle comme intrinsèquement perverse. Peu de subalternistes se satisferont de la réponse classique si souvent opposée à ce genre de constat, selon laquelle le réformisme libéral en milieu non occidental aboutit presque inéluctablement à des résultats partiels ou biaisés, générateurs de tensions et d'échecs souvent pires que le mal qu'il prétend corriger. Dipesh Chakrabarty exprimait avec vigueur en 1995 ce qu'un tel diagnostic a encore de commun avec les travers européocentriques de l'orientalisme : "Pourquoi la modernité serait-elle encore l'avenir qui nous attend plus de deux cents ans après avoir été introduite par l'impérialisme européen ? Combien de temps faut-il à un Indien pour devenir moderne? Une modernité pleine et entière [... serait] par définition quelque chose de bon, l'incarnation de la prospérité, du rationalisme. On ne dit jamais : ce que nous avons est tout ce que nous avons. On dit: ce que nous avons n'est qu'une mauvaise version de ce qui en soi est bon. Et on ajoute que nous avons encore du chemin à faire.[...] Passer du registre des lamentations à celui de l'ironie: voilà le déplacement qu'opère l'incrédulité face aux métarécits de l'Europe des Lumières. "Cette ironie de l'auteur vise entre autres la phrase inaugurale d'un livre de Sumit Sarkar (le meilleur manuel existant relatif à l'histoire de l'Inde à l'époque du nationalisme), où il est dit que l'Inde a traversé au cours du siècle écoulé la plus grande transition de son histoire, "une transition, cependant, qui est restée par bien des côtés gravement incomplète » ${ }^{24}$. Face au discours hégémonique de la modernité et du progrès, à cette "propagande de la raison", à cette "monomanie de l'imagination à l'œuvre chez le sujet savant, juge et maître qui sait toujours déjà ce qui est bon pour tout le monde avant même de s'en être assuré ", il faut, écrit Chakrabarty, "aller vers le subalterne", "le laisser mettre en question nos conceptions de l'universel »,

24. D. Chakrabarty 1995: 755. Le manuel en question est Sarkar 1983; mes italiques.

25. Chakrabarty, ibid. : 757 et 753. 
faire leur place à l'affectif, au religieux, à « ce que nous avons fini, en devenant modernes, par voir comme irrationnel ${ }^{25}$.

Le qualificatif de subalterne, dans cette perspective, n'est plus qu'un intitulé fédérateur symbolisant toutes les formes de différence culturelle qui survivent dans la situation coloniale, tous les domaines de la pensée et de l'expérience de la société dominée qui demeurent inaccessibles ou réfractaires à l'emprise autoritaire du rationalisme des Lumières, de la modernité occidentale, de l'État-nation : le privé ou le domestique face au public (c'est-à-dire le domaine politique, économique, administratif, technique où règne le pouvoir colonial), le religieux, l'artistique, l'affectif face au rationnel, le communautaire, le local, le marginal face à l'étatique et au national, et bien entendu le précolonial face à tout ce qui a suivi. Ce sont là, désormais, les domaines d'investigation électifs des Subaltern Studies. Tous sont également valorisés par opposition au modèle colonial moderne, du moment qu'ils désignent une différence, qu'ils se distinguent comme "incommensurables ", qu'ils peuvent faire figure d'antimodèles (pour reprendre un lexique postmoderniste qui figure maintenant largement chez nos auteurs). Un autre mot clé est le "fragment", antonyme polyvalent du souverain, de l'universel, de l'homogène, du continu, et qui s'applique particulièrement aux segments sociaux opprimés et réduits au silence par l'État-nation, dont le projet subalterniste vise à restituer la parole dissidente ${ }^{26}$. Par contamination, la structure des livres elle-même s'atomise, et les chapitres s'achèvent sans conclusion : «S'exprimer au nom du fragment ", écrit P. Chatterjee (1993 : 13), «c'est aussi produire, nul ne s'en étonnera, un discours qui est lui-même fragmentaire" (cf. Amin 1995). Enfin les sources interrogées sont de plus en plus souvent d'ordre littéraire. Cela s'explique certes en partie par le fait que les Subaltern Studies ont été placées dès l'origine par Ranajit Guha sous le signe de la critique textuelle, et aussi que les départements de littérature des universités américaines sont l'un des foyers de dissémination les plus actifs des questionnements épistémologiques qui inspirent aujourd'hui les auteurs de la série (Edward Said, rappelons-le, mais aussi Gayatri Spivak, un des membres les plus en vue du collectif, appartiennent à cette mouvance). Mais il faut voir là surtout l'effet d'un déplacement de l'attention des archives coloniales en langue anglaise vers la littérature imprimée en langues vernaculaires (A. Chakrabarty 1995 ; Ramachandra Guha 1996). Cette littérature périssable, dont la production était immensément dispersée, et qui n'a été que peu conservée ${ }^{27}$, ouvre en effet un champ d'investi-

26. Cf. Pandey 1992 (repris in Diouf 1999); Chatterjee 1993.

27. La "Vernacular Tracts Collection » de l'India Office Library (la structure qui abrite les archives coloniales de l'Inde à Londres) est un des conservatoires les plus utilisés de cette littérature. 
gation très prometteur à l'histoire culturelle. Ce champ n'était pas inconnu des chercheurs auparavant, mais ils sont de plus en plus nombreux à le fréquenter depuis une quinzaine d'années, bien qu'un maniement pertinent des différents niveaux de lecture que suppose l'utilisation historienne de ces sources exige des procédures critique très concertées. Évidemment, on s'éloigne un peu plus par ce biais de la considération exclusive du subalterne illettré, privé de parole, asservi aux élites de son propre peuple autant qu'au pouvoir colonial, qui était au centre du propos subalterniste initial. C'est avant tout d'identité culturelle qu'il s'agit désormais, le critère discriminant qui préside au choix de l'objet d'étude étant le degré d'immunité maintenu face à l'invasion de la modernité coloniale. Le sujet du dernier livre de D. Hardiman, membre fondateur du collectif, Feeding the Baniya (1996), qui traite avec maitrise de l'oppression des paysans par les usuriers dans l'Inde occidentale de l'époque coloniale, en apparaît presque incongru.

Les difficultés et les critiques soulevées par cette orientation sont à la fois d'ordre scientifique et d'ordre politique. Les subalternistes répudient toute approche de la culture et de l'action des subalternes qui « anthropologise » la différence culturelle en la référant à la grille interprétative universelle de la rationalité, de même qu'ils refusent une histoire qui situe et qualifie l'activité contestataire des subalternes comme marginale ou réactionnaire selon les critères du métarécit du progrès. La question, ajoute D.Chakrabar ty (1998), n'est pas tant de produire de la «bonne " histoire conforme aux discours de la méthode de Robin G. Collingwood, d'Edward H. Carr ou de Marc Bloch, que de la connaissance subversive. Sans sombrer dans l'irrationalisme, il faut tirer l'histoire vers sa limite, tâtonner vers des formes de démocratie que peut-être nous ne comprenons pas bien encore (Chakrabarty 1998). Les subalternistes seraient donc ainsi passés de la critique de l'historiographie élitiste dominante, coupable d'occulter le domaine de l'autonomie des subalternes, à la critique pure et simple de l'histoire en tant que discipline compromise avec les grands systèmes discursifs de la modernité. L'humanisme des Lumières étant dénoncé comme l'impensé du marxisme, la critique subalterniste de la modernité englobe la pensée de Marx, dont les analyses seraient imprégnées d'un discours d'émancipation qui offrit autrefois une justification à l'expansion coloniale de l'Europe et qui sert de nos jours à légitimer l'État-nation (Prakash 1994: 1489-1490). Ce sont là de grandes questions. Force est cependant de constater que les subalternistes font toujours de l'histoire, d'ailleurs le plus souvent avec talent, et qu'ils ne sont philosophes que d'occasion. Maniée sans précaution comme outil heuristique pour l'histoire, la valorisation systématique du précolonial ou de l'indi- 
gène par opposition au colonial risque évidemment de conduire au simplisme le plus caricatural, plus aisément encore que la réification de l'opposition élite/subalternes. Elle représente en un sens le négatif du cliché que l'historiographie coloniale a profondément enraciné dans les esprits, qui fait de la conquête européenne une césure radicale et un commencement dans tous les domaines de la vie indienne. Or, c'est précisément en s'efforçant de relativiser ce postulat pour progresser vers une appréciation plus nuancée des processus historiques que l'historiographie de l'Inde a fait ces vingt dernières années certaines de ses plus incontestables avancées.

Dans l'ordre politique, ce que dénoncent des critiques comme Sumit Sarkar, c'est le risque de dérive du nouveau cours de l'historiographie subalterniste dans le sens du néo-traditionalisme, du communautarisme, des idéologies identitaires, voire même de régression vers un indigénisme étroit, soupçon contre lequel les subalternistes eux-mêmes se défendent d'ailleurs vigoureusement. La question, assurément, mérite d'être posée s'agissant d'historiens qui valorisent, face à l'État colonial défini comme incarnation oppressive de la rationalité et de la modernité occidentales, des communautés indigènes conçues comme des entités culturelles indifférenciées, sans considération des inégalités et des rapports de pouvoir qu'elles recèlent ; quand le combat nationaliste pour les libertés tend à être présenté comme une conduite de compromission avec l'idéologie des oppresseurs; quand le fait d'avoir reçu une éducation moderne (supposant la pratique de l'anglais) est connoté négativement comme signe de subordination, etc. (Sarkar 1993, 1997 : 98, 106, 1999 : 315-321 ; Bahl 1997). La dénonciation de ce risque coïncide bien entendu avec une critique de gauche très générale du postmodernisme, qui est accusé de faire le lit des idéologies identitaires tout en évacuant comme mythologique l'idéologie de la lutte des classes, et elle rejoint le reproche courant qui lui est fait de conduire au relativisme. Mais elle se charge aussi, dans la conjoncture politique spécifique de l'Inde actuelle, de connotations beaucoup plus lourdes de sens. L'Inde n'a pas encore été gagnée par le désintérêt des sociétés nanties pour les luttes partisanes collectives, et les grands mouvements revendicatifs y restent une composante fondamentale de la vie politique. Et surtout la montée en puissance puis l'accession au pouvoir des nationalistes hindous (qui dénoncent l'État-nation libéral et laïc comme une importation occidentale), l'exacerbation des antagonismes inter-communautaires, l'ébranlement des principes du "sécularisme" officiel de l'État indien et l'érosion de ses bases légales et institutionnelles, donnent une résonance d'une particulière gravité à toute démarche intellectuelle susceptible de contribuer à légitimer l'inacceptable, ou en tout cas d'affaiblir la position de ceux qui en combattent la menace. 
Nul ne peut certes soupçonner les auteurs subalternistes de vouloir appuyer l'intolérance religieuse, le statu quo social, et encore moins la violence. La plupart restent en profondeur des intellectuels radicaux. Ils ont d'ailleurs produit des études souvent éclairantes sur la genèse idéologique du nationalisme hindou et des antagonismes entre confessions. C'est le cas de Partha Chatterjee, qui a montré tout ce que la rhétorique de l'extrémisme hindou emprunte à l'imaginaire nationaliste de la nation, définie comme une entité naturelle homogène. C'est encore plus nettement le cas de Gyan Pandey, qui a produit des réflexions très poussées sur les origines coloniales et nationalistes du communalisme et sur la représentation des violences interconfessionnelles dans l'historiographie (laquelle occulterait la responsabilité de la modernité capitaliste et de l'État-nation dans le déclin de l'harmonie intercommunautaire précoloniale) (Chatterjee 1994 ; Pandey 1990, 1992 et 1994). Mais, face aux engagements qui s'imposent, ces auteurs sont aujourd'hui confrontés, dit Sarkar, aux apories d'un discours qui tourne en dérision toute référence à la raison et au progrès, qui détecte un risque totalitaire dans toute forme de résistance autre que fragmentaire et dépourvue de visée réformatrice d'ensemble, et qui se nourrit de la contemplation esthétique des différences et de la nostalgie de l'authenticité indigène disparue (Sarkar 1997 : 107-108, 1999 : 321-322).

Dans un article engagé paru en 1992, les historiens indianistes Rosalind O’Hanlon et David Washbrook fustigeaient déjà sévèrement dans le même esprit l'historiographie "post-orientaliste » des Tiers-Mondes en général et de l'Inde en particulier, définie comme un amalgame de culturalisme différentialiste, de critique foucaldienne du pouvoir et de délégitimation postmoderniste des métarécits ${ }^{28}$. Ils concluaient qu'on avait là affaire, en fait, au dernier avatar de l'éternelle mauvaise foi de l'Occident libéral expansionniste, qui sanctifie les libertés fondamentales à domicile tout en maintenant outre-mer des situations d'exploitation : sous couvert d'une posture critique censée restaurer l'Autre dans sa vérité culturelle et son autonomie, on le dépolitise et on l'enferme dans sa condition de dominé en récusant a priori toute idéologie d'émancipation comme un masque de l'oppression. Leur analyse amorçait donc une déconstruction de ce discours si habile à déconstruire le discours des autres. Ils y voyaient un effet de la «domestication » de la pensée poststructuraliste et postmoderniste française (Michel Foucault, Gilles Deleuze, Jean-François Lyotard, Jacques Derrida, Jean Baudrillard pour l'essentiel), adaptée et vidée de son contenu politique radical par la culture libérale des universités américaines, et infléchi dans un sens culturaliste parce que ce milieu est travaillé en profondeur par le problème de l'auto- 
représentation, de la coexistence et des conflits des multiples minorités qui le composent. Cette analyse renvoie évidemment à la question de la diaspora nord-américaine des intellectuels issus d'Asie du Sud dont il est question dans ce numéro. Sarkar rejoint cette préoccupation en soulignant le rôle joué par certains intellectuels du Sud établis en Occident dans l'apparition de la "postcolonialité » parmi les thèmes-vedettes de ce nouveau conformisme intellectuel, et en suggérant aussi que professer ces thèses est particulièrement payant aux États-Unis en termes de carrière universitaire (Sarkar $1997: 85,1999: 310)$.

Il va de soi qu'on ne liquidera pas les questions importantes posées par ce courant de pensée avec un sarcasme relatif à ses conditions d'émergence. La conviction d'opérer dans le camp des précurseurs, la vigueur des attaques reçues, la mise en place d'une nouvelle orthodoxie ont certes parfois amené les néo-subalternistes, comme il est inévitable, à proférer des anathèmes, à avancer des thèses hasardeuses, à échafauder des lignes de défense hâtives. Il est cependant dans l'ordre des choses qu'on retrouve, dans les sciences sociales d'un pays du Sud qui s'annonce comme l'une des grandes puissances du XXI ${ }^{\text {e }}$ siècle, un écho de la mise en cause de l'humanisme des Lumières engendrée par les tragédies collectives $\mathrm{du} \mathrm{XX}^{\mathrm{e}}$, sous la forme justifiée d'une critique de l'impérialisme des systèmes doctrinaux et des appareils de pouvoir dans lesquels il s'est incarné. Que cet écho soit produit ou répercuté en partie par des intellectuels expatriés ne peut davantage étonner à l'âge de la mondialisation. S'ils rejettent maintenant pour la plupart le corps de doctrine du grand récit marxiste orthodoxe, les subalternistes n'en restent pas moins fidèles à ce qui, chez Marx, va dans le sens du rejet du totalitarisme, à l'esprit de critique sociale radicale qui lui vient des Lumières, comme l'est au demeurant l'une de leurs figures tutélaires, Derrida, dans ses Spectres de Marx (1993). Le vrai problème est ailleurs, dans le remplacement des idéologies universalistes de l'émancipation par des moralités communautaires, dans la tendance à l'esthétisation des différences, dans les implications obscurantistes d'un antimodernisme parfois trop emporté, attitudes intellectuelles qui peuvent servir à justifier, dans un contexte politique et social aussi troublé que celui de l'Inde, des dérives dont personne ne veut. Cela étant, il ne faut pas surestimer l'impact des Subaltern Studies sur la classe intellectuelle ni sur les luttes sociales. D'une part, la masse de l'historiographie indienne demeure anticoloniale au sens nationaliste classique. D'autre part, les institutions de l'État démocratique né de l'indépendance et les idéaux de la modernité restent la base de ressource principale des mouvements sociaux. Peut-être faut-il en voir une illustration symbolique dans la réaction manifestée en 
1997 par un intellectuel tamoul, à l'occasion d'une rencontre organisée autour des Subaltern Studies dans la petite ville de Kumbakonam dans l'extrême sud de l'Inde. Il critiquait le projet subalterniste, entreprise dominée selon lui par des Brahmanes du Bengale, en tant qu'il ignore l'exclusivisme du sentiment de caste comme facteur constitutif de la subalternité en Inde, et ajoutait qu'un courant de recherche qui répudie la modernité ne peut mener en Inde qu’à un néo-brahmanisme... (Pandian 1997). Mais il y a place encore pour des approfondissements, des clarifications, voire des corrections de trajectoire. Lors de la sixième Subaltern Studies Conference qui s'est tenue à Lucknow en 1999 sous l'intitulé significatif "Fractured Societies, Fractured Histories", Partha Chatterjee déclarait que la catégorisation élite/subalternes ne se substitue pas à la théorie des classes mais la complète, parce qu'elle rend mieux compte des contradictions propres aux formations sociales du Tiers Monde et des caractères spécifiques de leur transition (Dhar \& Verma 1999: 1094). Un tel propos semble maintenir le cap d'une critique sans dogmatisme du champ social. Affaire à suivre...

MOTS CLÉS/KEYWORDS: histoire sociale/social history - Asie du Sud/South Asia - orientalisme/orientalism - postcolonialitélpostcoloniality - postmodernismelpostmodernism.

BIBLIOGRAPHIE

Amin, Shahid

1984 "Gandhi as Mahatma: Gorakhpur

District, Eastern UP 1921-1922», in

Ranajit Guha, ed., Subaltern Studies III.

Delhi, Oxford University Press : 1-61.

1995 Event, Memory, Metaphor: Chauri Chaura 1922-1992. Delhi, Oxford University Press.

Anderson, Benedict

1983 Imagined Communities: Reflections on the Origin and Spread of Nationalism.

London, Verso.

Arnold, David

1984 "Gramsci and Peasant Subalternity in India ", Journal of Peasant Studies 11(4) :

155-177.

Bahl,Vinay

1997 "Relevance (or Irrelevance) of

Subaltern Studies ", Economic and Political

Weekly, 7 June : 1933-1944.
Bhattacharya, Sabyasachi

1983 "History from Below ", Social

Scientist 119.

Brass, Tom

1993 "A-way with their World : Rural Labourers through the Postmodern Prism ", Economic and Political Weekly, 5 June: 1162-1168.

Chakrabarty, Anita 1995 "Writing History", Economic and Political Weekly, 23 Dec. : 3320.

Chakrabarty, Dipesh

1985 "Invitation to a Dialogue », in Ranajit Guha, ed., Subaltern Studies IV. Delhi, Oxford University Press : 364-376. 1991 «History as Critique and Critique(s) of History ", Economic and Political Weekly, 14 Sept. : 2162-2166. 
1992 "Postcoloniality and the Artifice of History: Who Speaks for the "Indian" Pasts ", Representations 37 : 1-26.

1995 «Radical Histories and Question of Enlightenment Rationalism : Some Recent Critiques of Subaltern Studies ", Economic and Political Weekly, 8 Apr. : 751-759.

1998 "Minority Histories, Subaltern Pasts ", Economic and Political Weekly, 28 Feb. : 473-479.

\section{Chatterjee, Partha}

1986 Nationalist Thought and the Colonial World: A Derivative Discourse? Delhi, Oxford University Press - London, Zed Books.

1988 "For an Indian History of Peasant Struggle ", Social Scientist 16 (11) : 3-17. 1993 The Nation and its Fragments: Colonial and Postcolonial Histories.

Princeton, Princeton University Press Delhi, Oxford University Press.

1994 "Claims on the Past: The Genealogy of Modern Historiography in Bengal ", in David Arnold \& David Hardiman, eds, Subaltern Studies VIII : Essays in Honour of Ranajit Guha. Delhi, Oxford University Press : 1-49.

\section{Cohn, Bernard S.}

1985 "The Command of Language and the Language of Command ", in Ranajit Guha, ed., Subaltern Studies IV. Delhi, Oxford University Press : 276-329.

Derrida, Jacques

1993 Spectres de Marx. L'État de la dette, le travail du deuil et la nouvelle Internationale. Paris, Galilée.

\section{Dhar, Hiranmay \& Roop Reekha Verma}

1999 «Fractured Societies, Fractured Histories ", Economic and Political Weekly, 8 May: 1094-1097.

Diouf, Mamadou, ed. 1999 L'historiographie indienne en débat. Colonialisme, nationalisme et sociétés postcoloniales. Paris-Amsterdam, Karthala-Sephis.

\section{Foucault, Michel (présenté par)}

1973 Moi, Pierre Rivière, ayant égorgé ma mère, ma soeur et mon frère... Paris,

\section{Guha, Ranajit}

1982 "On Some Aspects of the Historiography of Colonial India ", in Ranajit Guha, ed., Subaltern Studies I. Delhi, Oxford University Press : 1-9.

1983a Elementary Aspects of Peasant Insurgency in Colonial India. Delhi, Oxford University Press.

1983b "The Prose of CounterInsurgency ", in Ranajit Guha, ed., Subaltern Studies II. Delhi Oxford University Press : 1-42.

1989 «Dominance without Hegemony and its Historiography ", in Ranajit Guha, ed., Subaltern Studies VI. Delhi, Oxford University Press : 210-309.

Guha, Ranajit \& Gayatri Spivak, eds 1988 Selected Subaltern Studies. New York, Oxford University Press.

\section{Guha, Ramachandra}

1996 «Beyond Bhadralok and Bankim Studies ", Economic and Political Weekly, 24 Feb. : 495-496.

\section{Hardiman, David}

1986 " "Subaltern Studies" at Crossroads", Economic and Political Weekly, 15 Feb. : 288290.

1987 The Coming of the Devi: Adivasi Assertion in Western India. Delhi, Oxford University Press.

1996 Feeding the Baniya: Peasants and Usurers in Western India. Delhi, Oxford University Press.

\section{Henningham, Stephen}

1983 "Quit India in Bihar and the Eastern United Provinces : the Dual Revolt ", in Ranajit Guha, ed., Subaltern Studies II. Delhi, Oxford University Press. 
Mukherjee, Mridula
1988 «Peasant Resistance and Peasant

Consciousness in Colonial India :

"Subalterns" and Beyond ", Economic and

Political Weekly, 8 Oct. et 15 Oct. : 2109 -

2120 et 2174-2185.

\section{O'Hanlon, Rosalind}

1988 «Recovering the Subject: Subaltern Studies and Histories of Resistance in Colonial South Asia ", Modern Asian Studies 22 (1) : 189-224.

\section{O'Hanlon, R. \& David Washbrook}

1992 "After Orientalism: Culture, Criticism and Politics in the Third World", Comparative Studies in Society and History 34 (1) : 141-167.

\section{Pandey, Gyanendra}

1990 The Construction of Communalism in Colonial North India. Delhi, Oxford University Press.

1992 «In Defence of the Fragment:

Writing about Hindu-Muslim Riots in India Today", Representations 37 : 27-55.

1994 "The Prose of Otherness ", in David Arnold \& David Hardiman, eds, Subaltern Studies VIII : Essays in Honour of Ranajit Guha. Delhi, Oxford University Press : 188225.

\section{Pandian, M. S. S.}

1997 "Kumbakonam Encounters Subaltern Studies ", Economic and Political Weekly, 22 Feb. : 398-399.

\section{Prakash, Gyan}

1990a Bonded Histories: Genealogies of Labor Servitude in Colonial India.

Cambridge, Cambridge University Press.

1990b «Writing Post-Orientalist Histories of the Third World : Perspectives from Indian Historiography ", Comparative Studies in Society and History 32 (2) : 383-408.
1992 "Can the "Subaltern" Ride? A Reply to O'Hanlon and Washbrook", Comparative Studies in Society and History 34 (1) : 168-184.

1994 «Subaltern Studies as Postcolonial Criticism ", American Historical Review 99 (5) : 1475-1490.

Sarkar, Sumit

1983a Modern India 1885-1947. Delhi, Macmillan-India.

1983b "Popular" Movements and "Middle Class" Leadership in Late Colonial India: Perspectives and Problems of a "History from Below". Calcutta, K. P. Bagchi/Centre for Studies in Social Sciences.

1993 "The Fascism of the Sangh Parivar", Economic and Political Weekly, 30 Jan. : 163167.

1997 "The Decline of the Subaltern in Subaltern Studies ", in Writing Social History. Delhi, Oxford University Press : 82108.

1999 "Postmodernism and the Writing of History", Studies in History 15 (2) : 293322.

\section{Spivak, Gayatri Chakravorty}

1985 «Subaltern Studies: Deconstructing Historiography ", in Ranajit Guha, ed., Subaltern Studies IV. Delhi, Oxford University Press : 330-363.

\section{Stein, Burton}

1985 "Notes on "Peasant Insurgency" in Colonial Mysore : Event and Process ", South Asia Research 5 (1) : 1-17.

\section{Thompson, Edward Palmer}

1988 La formation de la classe ouvrière anglaise. Paris, Le Seuil-EHESS-Gallimard. (Trad. de The Making of the English Working Class. London, 1963.) 
Jacques Pouchepadass, Les Subaltern Studies ou la critique postcoloniale de la modernité. — La série indienne en cours de publication, depuis bientôt vingt ans, sous le titre Subaltern Studies incarne un courant intellectuel issu de l'histoire sociale radicale qui est devenu le point d'entrée principal des idées postmodernistes en Asie du Sud. La mise en cause du rationalisme des Lumières, des idéologies du progrès et de l'État-nation est ici relayée par des intellectuels du Sud, dont bon nombre travaillent d'ailleurs dans des universités nord-américaines, et se nourrit du discours critique de la postcolonialité. Son audience, à cet égard, commence à s'étendre à d'autres continents du monde anciennement colonisé.
Jacques Pouchepadass, Subaltern Studies, or the Postcolonial Critique of Modernity. ted essays whose publication began almost twenty years ago, was born under the auspices of radical social history, and has now become a major entry point of postmodernist ideas in the field of South Asian social sciences. The critique of Enlightenment rationality, of the metanarratives of progress and of the nation-State which underlies the work of its contributors, mostly South Asian social scientists not unfrequently associated with North American universities, is intimately correlated with the discourse of postcoloniality. As a result, the audience of the series is now expanding in other areas of the formerly colonized world. 